\title{
Controlled release of optimized electroporation enhances the transdermal efficiency of sinomenine hydrochloride for treating arthritis in vitro and in clinic
}

\author{
This article was published in the following Dove Press journal: \\ Drug Design, Development and Therapy \\ 15 June 2017 \\ Number of times this article has been viewed
}

\author{
Shun Feng, ${ }^{1}, *$ Lijun Zhu, ${ }^{1, *}$ \\ Zhisheng Huang, ${ }^{2}$ Haojia \\ Wang,' Hong Li,' Hua \\ Zhou, ${ }^{3}$ Linlin Lu, 'Ying \\ Wang,' Zhongqiu Liu, ${ }^{1,3}$ \\ Liang Liu' ${ }^{1,3}$ \\ 'International Institute for \\ Translational Chinese Medicine, \\ Guangzhou University of Chinese \\ Medicine, Guangzhou, Guangdong, \\ ${ }^{2}$ Department of Acupuncture and \\ Rehabilitation, Guangzhou Hospital \\ of Integrated Traditional Chinese \\ and Western Medicine, Guangzhou, \\ Guangdong, ${ }^{3}$ State Key Laboratory of \\ Quality Research in Chinese Medicine, \\ Macau University of Science and \\ Technology, Macau, People's Republic \\ of China \\ *These authors contributed equally \\ to this work
}

\begin{abstract}
Sinomenine hydrochloride $(\mathrm{SH})$ is an ideal drug for the treatment of rheumatoid arthritis and osteoarthritis. However, high plasma concentration of systemically administered SH can release histamine, which can cause rash and gastrointestinal side effects. Topical delivery can increase SH concentration in the synovial fluid without high plasma level, thus minimizing systemic side effects. However, passive diffusion of SH was found to be inefficient because of the presence of the stratum corneum layer. Therefore, an effective method is required to compensate for the low efficiency of SH passive diffusion. In this study, transdermal experiments in vitro and clinical tests were utilized to explore the optimized parameters for electroporation of topical delivery for SH. Fluorescence experiment and hematoxylin and eosin staining analysis were performed to reveal the mechanism by which electroporation promoted permeation. In vitro, optimized electroporation parameters were $3 \mathrm{KHz}$, exponential waveform, and intensity 10 . Using these parameters, transdermal permeation of SH was increased by 1.9-10.1 fold in mice skin and by 1.6-47.1 fold in miniature pig skin compared with passive diffusion. After the electroporation stimulation, the intercellular intervals and epidermal cracks in the skin increased. In clinical tests, SH concentration in synovial fluid was $20.84 \mathrm{ng} / \mathrm{mL}$ after treatment with electroporation. Therefore, electroporation with optimized parameters could significantly enhance transdermal permeation of SH. The mechanism by which electroporation promoted permeation was that the electronic pulses made the skin structure looser. To summarize, electroporation may be an effective complementary method for transdermal permeation of SH. The controlled release of electroporation may be a promising clinical method for transdermal drug administration.
\end{abstract}

Keywords: electroporation, sinomenine hydrochloride, transdermal drug delivery, arthritis

\section{Introduction}

Rheumatoid arthritis (RA) is a refractory and chronic inflammatory disease, while osteoarthritis (OA) is the most common disorder of the locomotor system. RA and OA can damage joint function, induce joint injury and arthromeningitis, and lead to premature death. ${ }^{1}$ Reports have shown that approximately $1 \%$ of the world population is suffering from RA and that OA affects mainly the elderly. ${ }^{2,3}$ Nonsteroidal anti-inflammatory drugs (NSAIDs) have been reported to be widely used in RA and OA therapy. ${ }^{4,5}$ However, NSAIDs can induce renal and gastrointestinal toxicities because they inhibit prostaglandin synthesis, thus limiting the clinical application of NSAIDs. ${ }^{6}$ Among the side effects, NSAID-induced enteropathy could increase small
Correspondence: Zhongqiu Liu; Liang Liu No 232, Waihuandong Road, Guangzhou University of Chinese Medicine, Guangzhou Higher Education Mega Center, Panyu District, Guangzhou, Guangdong, 5 10006, People's Republic of China

Tel +86203935806I

Fax +86203935 807I

Email liuzq@gzucm.edu.cn; Iliu@must.edu.mo
Drug Design, Development and Therapy 2017: | I 1737-1752

1737

Dovepress f in 0

http://dx.doi.org/10.21471PDDTS136313 (c) (1) \& $\odot 2017$ Feng et al. This work is published and licensed by Dove Medical Press Limited. The full terms of this license are available at https://www.dovepress.com/terms.php cc) hereby accept the Terms. Non-commercial uses of the work are permitted without any further permisision from Dove Medical Press Limited, provided the work is properly atributed. For permission for commercial use of this work, please see paragraphs 4.2 and 5 of our Terms (https://www.dovepress.com/terms.php). 
intestine bleeding and ulcers. ${ }^{7}$ Thereby, choosing a drug to reduce RA and OA symptoms and prevent future adverse effects is necessary.

Sinomenine $\left(\mathrm{C}_{19} \mathrm{H}_{23} \mathrm{NO}_{4}\right)$, extracted from the stems and roots of Sinomenium acutum, is a kind of pharmaceutically active alkaloid with few side effects. ${ }^{8}$ Previous pharmacological studies have demonstrated that sinomenine has analgesic, ${ }^{9}$ anti-inflammatory, ${ }^{10}$ cartilage protection, ${ }^{11}$ and immune suppression effects. ${ }^{12}$ For decades, sinomenine has been widely used to treat RA and OA in China. ${ }^{13}$ Compared with NSAIDs, sinomenine is more effective in amelioration of morning stiffness, painful joints, and erythrocyte sedimentation rate, with less adverse effects on the digestive system. ${ }^{14}$ Therefore, sinomenine is a valuable remedy to treat RA and $\mathrm{OA}$ in clinical practice.

Sinomenine hydrochloride (SH, Figure 1) is designed to provide better efficacy with daily application. ${ }^{15} \mathrm{SH}$ tablets and injections have been used effectively for RA and OA therapy. The anti-RA and anti-OA effect of SH might be closely connected with its antiproliferative effect on inflammatory indexes on articular tissue and intervention of gristle degeneration and cartilage cell apoptosis. ${ }^{16,17}$ Hence, the articular tissue is the primary therapeutic target of SH. SH concentration within the articular tissues, such as synovial fluid (SF) or synovial membrane, is important. Nevertheless, SH tablets and injections are systemically administered. Moreover, the concentration of systemically administered $\mathrm{SH}$ in plasma was much higher compared with that in SF. ${ }^{18}$ Therefore, comparatively higher plasma concentration is necessary for achieving adequate amounts of SH in SF via systemic administration. Previous studies have demonstrated that high plasma concentration of $\mathrm{SH}$ will lead to potent release of histamine in association with the degranulation of mast cells in mammalian tissues, ${ }^{19}$ and this could cause rash and gastrointestinal side effects (eg, nausea, diarrhea, gastralgia, and occasionally vomiting). Research has also found that the adverse effects of SH appearing in some animals after dosing might be related to high plasma concentration of rapid $\mathrm{SH}$ distribution to the internal organs. ${ }^{20}$ Undoubtedly, topical transdermal drug delivery targeting the joint tissue is a promising therapeutic method for RA and OA patients. On one hand, it decreases drug plasma concentration and SH distribution into the internal organs, which could alleviate side effects. On the other hand, it increases drug concentration in SF, which could enhance drug efficiency. Concurrently, transdermal drug delivery might enable the drug to bypass the first pass effect (oral administration) and might provide relatively steady drug concentration in SF for long periods of time. ${ }^{21}$ Although transdermal delivery of $\mathrm{SH}$ has been reported, the application of the transdermal delivery system is often limited due to the stratum corneum (SC) layer, which is the primary barrier of the outermost skin layer. ${ }^{22}$ The transdermal delivery did not enable the passive diffusion of $\mathrm{SH}$ into the skin, thereby reducing $\mathrm{SH}$ efficacy.

At present, some physical means, such as microneedles, ${ }^{23}$ ionophoresis, ${ }^{24}$ sonophoresis, ${ }^{25}$ and electroporation, ${ }^{26}$ have been utilized to enhance transdermal drug delivery. Electroporation has been considered as an efficient physical technique of promoting skin permeability of small molecules and biomacromolecules. ${ }^{18,27}$ Electroporation can compensate for the defects of passive diffusion and significantly increase transdermal efficiency by using a series of short, high-voltage electric pulses. ${ }^{28,29}$ Furthermore, electroporation uses only a small instrument, is safe, and is convenient for self-administration by patients.

However, the mechanism by which electroporation improves transdermal efficiency is not clearly understood. For example, why and how could electroporation enhance permeation is yet to be determined. In this study, fluorescence method and HE staining were chosen to explore the transdermal enhancement mechanism of electroporation. The selection of appropriate parameters of electroporation is one of the keys to high transdermal enhancement effect of electroporation. Three parameters, namely, pulse frequency, pulse waveform, and pulse intensity, of electroporation were selected as the typical parameters to explore the above relationship. The transdermal enhancement effect of electroporation was confirmed by the using optimized parameters for electroporation, facilitating clinically transdermal permeation tests. Highly efficient transdermal delivery by using the optimized parameters of electroporation is recommended to enhance $\mathrm{SH}$ transdermal administration method in future clinical application.

\section{Materials and methods Materials}

SH solution ( $25 \mathrm{mg} / \mathrm{mL}$, injection) was provided by Zhengqing Pharmaceutical Group Co., Ltd. (Hunan, People's Republic of China). SH (Standard substance) was purchased from Dalian Meilun Biotech Co., Ltd. (Dalian, People's Republic of China). $\mathrm{CaCl}_{2}, \mathrm{KCl}, \mathrm{NaCl}$, and $\mathrm{NaHCO}_{3}$ were purchased from Sigma-Aldrich (St Louis, MO, USA). Testosterone, used as internal standard (IS), was purchased from Nacalai Tesque Company (Kyoto, Japan). Sodium fluorescein 

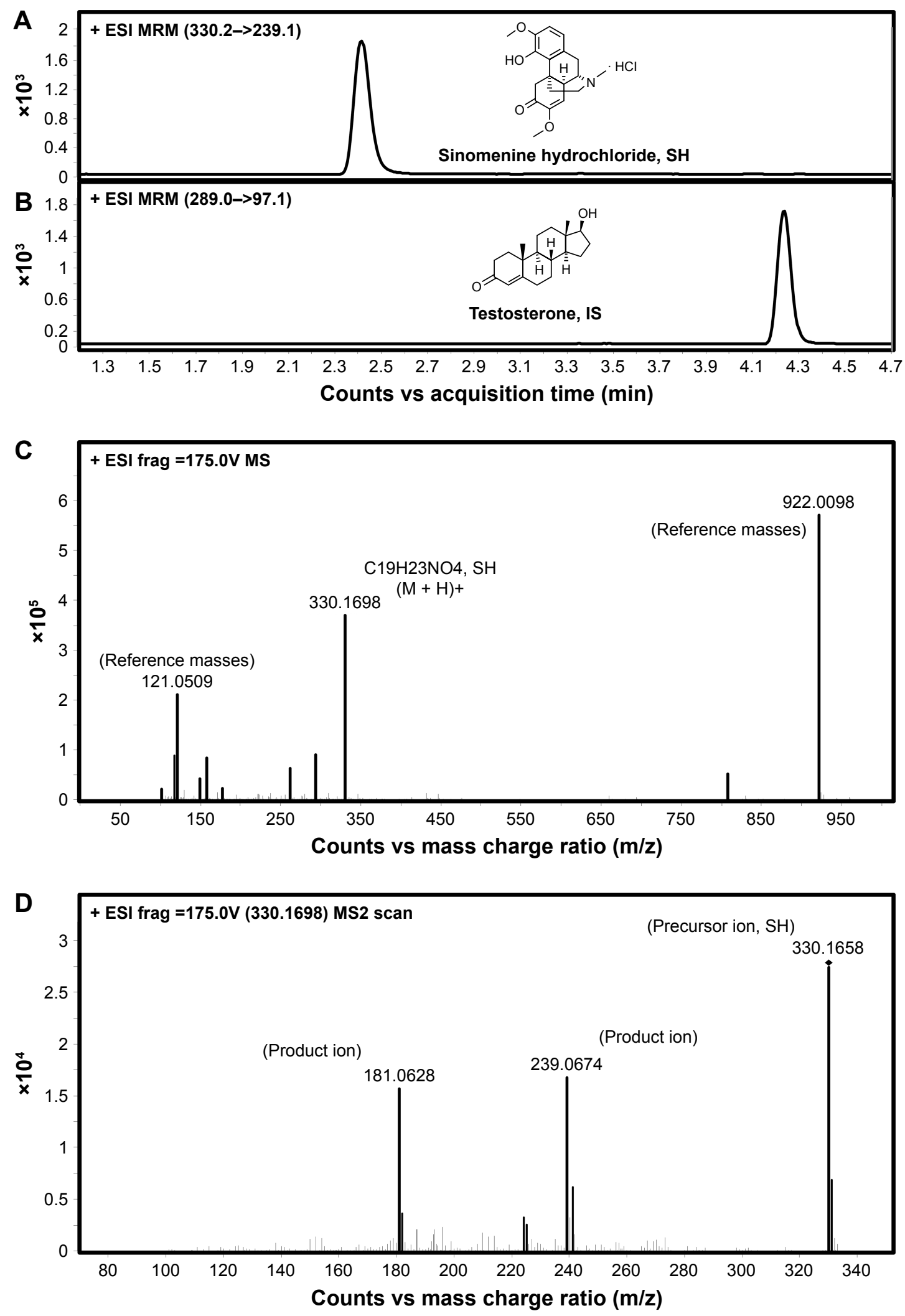

Figure I UHPLC-MS/MS method for analysis of SH in Ringer's solution and SF.

Notes: The compound-dependent parameters were in MRM method. (A) The transition of SH was 330.2/239.1. (B) The transition of testosterone (IS) was 289.0/97.I. (C) and (D) were the MS spectra and the MS/MS spectra of SH, respectively.

Abbreviations: $\mathrm{SH}$, sinomenine hydrochloride; IS, internal standard; MRM, multiple reaction monitoring; MS, mass; ESI, electron spray ionization; SF, synovial fluid; UHPLC, ultra high performance liquid chromatography. 
(NaFluo) was from GBCBIO Biological Technology Co., Ltd. (Guangzhou, People's Republic of China). Urethane was purchased from Sinopharm Chemical Reagent Co., Ltd. (Beijing, People's Republic of China). The water used for the ultra-high performance liquid chromatography-mass spectroscopy/mass spectroscopy (UHPLC-MS/MS) analysis was obtained from a Milli-Q water purification system produced by Millipore (Billerica, MA, USA). HPLC-grade acetonitrile, methanol, and formic acid were acquired from Merck (Darmstadt, Germany). Other reagents were of analytical grade.

\section{Animals}

All experiments were performed in accordance with the National Institutes of Health Guide for the Care and Use of Laboratory Animals (1996). The animal protocol used in the study was approved by the Institutional Animal Care and Use Committee of the Guangzhou University of Chinese Medicine. Male Kunming mice (weighing 18-22 g) were used in the current experiments. All mice were purchased from the Laboratory Animal Center of Guangzhou University of Chinese Medicine (Guangzhou, People's Republic of China) and were raised in the animal houses of the Laboratory Animal Center of Guangzhou University of Chinese Medicine. Four mice were housed per cage in a clean animal cabinet with ad libitum access to nutritionally adequate food and uncontaminated drinking water throughout the experimental periods. The mice were housed in an animal cabinet with unidirectional air flow under $40 \%-70 \%$ relative humidity, $20^{\circ} \mathrm{C}-24^{\circ} \mathrm{C}$, and $12 \mathrm{~h}$ light/dark cycle. The abdominal skins of Bama miniature pigs (weighing $13 \mathrm{~kg}$ ) were purchased from and prepared at Taizhou Taihe Biotech Co., Ltd. (Taizhou, People's Republic of China). All animals were killed by cervical luxation after anesthesia with an intraperitoneal injection of urethane $(50 \%, \mathrm{w} / \mathrm{v})$.

\section{Skin preparation}

The back skins of mice and the abdominal skins of Bama miniature pig were cut using surgical instruments and washed with Ringer's solution or normal saline. The skins were divided into $3 \mathrm{~cm}^{2}$ size and stored at $-80^{\circ} \mathrm{C}$. The pieces of skins were used within 3 months. The skins were thawed at room temperature and then soaked in Ringer's solution ( $\mathrm{pH}$ 7.4) for $1 \mathrm{~h}$ before transdermal experiments.

\section{Passive diffusion study}

Percutaneous experiments in vitro were done on YB-P6 intelligent transdermal diffusion instrument, which was purchased from Tianjin Pharmacopoeia Standard Instrument Factory (Tianjin, People's Republic of China), with the perpendicular Franz-type diffusion cells. The diffusion area was $1.96 \mathrm{~cm}^{2}$. The pieces of skin tissue were sandwiched between the receiver and donor compartments, and the epidermal side was facing up (Figure 2). Diffusion was continued for $8 \mathrm{~h}$ at $37^{\circ} \mathrm{C} \pm 0.5^{\circ} \mathrm{C} .{ }^{30} \mathrm{~A}$ total of $2 \mathrm{~mL} \mathrm{SH}$ solution was added to the donor compartment. The mouths of donor compartments were covered with parafilm (Bemis Company, Oshkosh, USA) to avoid liquid evaporation. The receiver compartments were filled with 17 mL Ringer's solution ( $\mathrm{pH} 7.4$ ) and stirred at $400 \mathrm{rpm}$. The samples $(3 \mathrm{~mL})$ were extracted from the receiver compartments for UHPLC-MS/MS analysis and then rapidly replaced with the same quantity $(3 \mathrm{~mL})$ of fresh Ringer's solution at $37^{\circ} \mathrm{C} \pm 0.5^{\circ} \mathrm{C}$ at $1,2,3,4,6$, and 8 h. Each experiment was repeated six times.

\section{Electroporation study}

An electroporation system (Yuandao Digital Electronics Co., Ltd., Shenzhen, People's Republic of China) was used to provide a series of electronic pulses. The electrode components, which were made of metal and shaped like a small stick, were placed in the donor and the receptor compartments (Figure 2). Other procedures were the same as described in "Passive diffusion study" section.

\section{Transdermal enhancement regulation with different electroporation parameters and different drug concentrations}

The relationship between the different frequency, waveform, and intensity of electroporation and the amount of SH that permeated in the in vitro transdermal experiments (mice and miniature pig skins) were studied under the fixed drug concentration.

To study the amount of $\mathrm{SH}$ that permeated at different drug concentrations, in vitro transdermal experiments (mice and miniature pig skins) were performed using fixed parameters of electroporation.

\section{Transdermal enhancement mechanism of electroporation}

\section{Hematoxylin and eosin staining}

Transdermal permeation experiments were done in vitro. Then, the mice and miniature pig skins were immediately collected and soaked in $4 \%$ paraformaldehyde solution for $24 \mathrm{~h}$ at $4^{\circ} \mathrm{C}$. The skin was then dehydrated using gradient alcohol and xylene, and then embedded in paraffin according to standard processes. Serial $5 \mu \mathrm{m}$-thick sections of skins were 


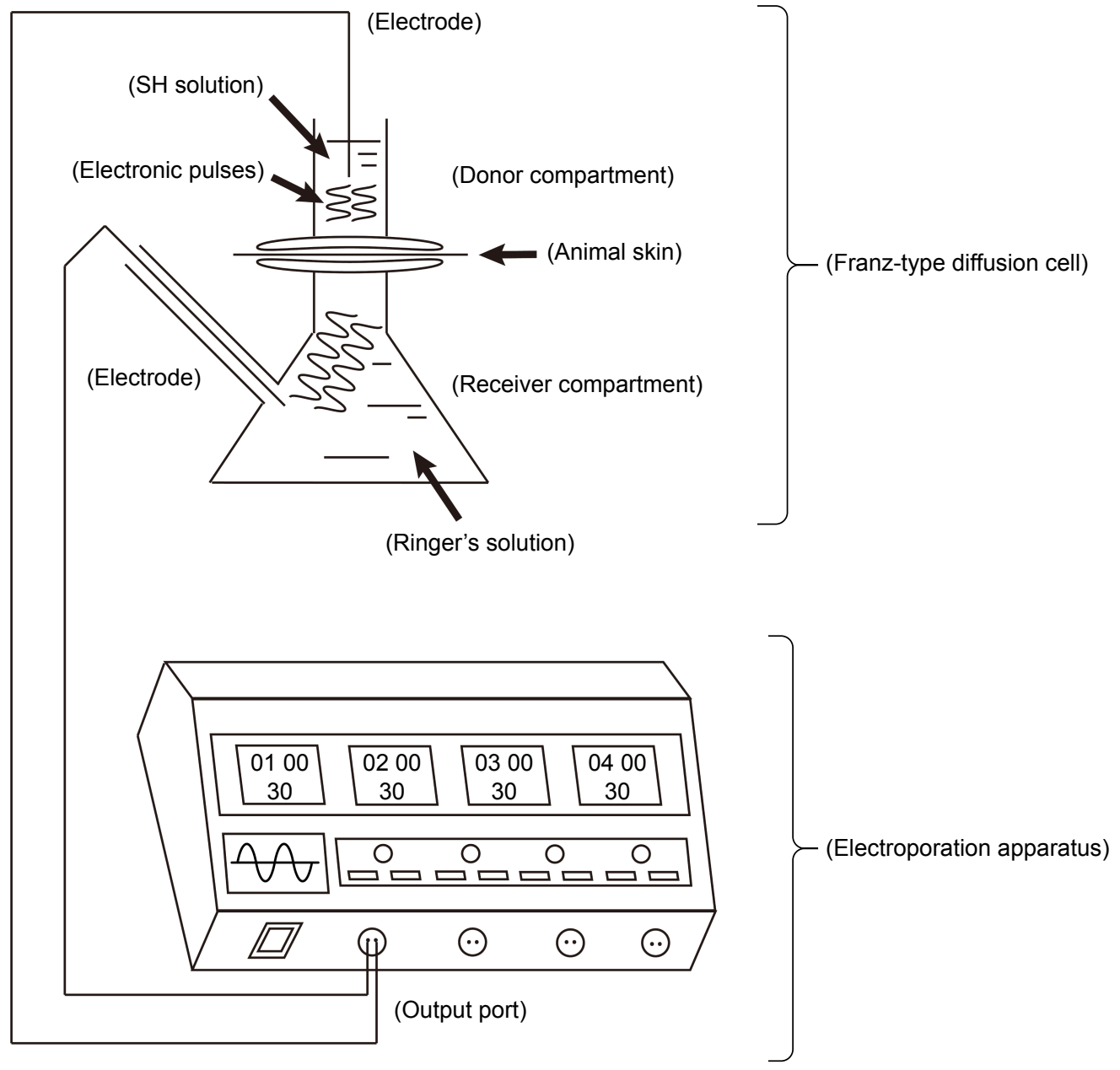

Figure 2 Flow diagram of transdermal experiment in vitro.

Notes: The skin tissues of mice and miniature pig were fixed between the donor and receiver compartments. Diffusion was continued for $8 \mathrm{~h}$ at $37^{\circ} \mathrm{C} \pm 0.5^{\circ} \mathrm{C}$. Abbreviation: $\mathrm{SH}$, sinomenine hydrochloride.

obtained using a Leica RM2235 microtome (Oberkochen, Germany). The histological properties of the skin were analyzed using hematoxylin and eosin (HE) staining and were observed with a Leica DM750 Microscopy.

\section{Transdermal permeation experiments of sodium fluorescein}

NaFluo (2 mg) was added to Ringer's solution $(2 \mathrm{~mL})$ and continually vortexed until it dissolved. Transdermal permeation experiments (on mice and miniature pig skins) were done using the NaFluo aqueous solution with or without electroporation following the method described in "Passive diffusion study" Section. ${ }^{31}$ Then, the skins were immediately collected and frozen in liquid nitrogen. The skins were then cut using a freezing microtome (Leica CM1860 UV, Leica Camera AG, Solms, Germany) into $5 \mu \mathrm{m}$-thick sections. The sections were washed thrice with Ringer's solution ( $\mathrm{pH}$ 7.4) to wash off free NaFluo and observed with a Leica TSC
SP8 Laser Confocal Microscopy (Oberkochen, Germany). The conditions were as follows: $488 \mathrm{~nm}$ excitation wavelength, $515 \mathrm{~nm}$ emission wavelength, 0 to 255 fluorescence intensity. ${ }^{32}$

\section{Transdermal treatment of SH with optimized electroporation in clinical tests} Patients and study design

The clinical tests were done at Guangzhou Hospital of Integrated Traditional Chinese and Western Medicine. These tests did not change the original treatment plan of patients. All patients in the tests were diagnosed with arthritic diseases (RA or OA) according to a detailed medical history for knee pain, a comprehensive physical exam, and imaging studies. The inclusion criteria were as follows: male or female $>40$ years old; presence of knee inflammation, knee pain, or any other symptom connected with knee arthritis diseases; and Kellgren-Lawrence radiological grade I-III. ${ }^{33}$ 
Meanwhile, the exclusion criteria were as follows: presence of severe cardiovascular disease, immunodepression, coagulopathy, liver disease, infection, and hematological disease; and pregnancy. In patients with bilateral arthritis, only the knee that showed more advanced disease was considered. The protocol was approved by Guangzhou Hospital of Integrated Traditional Chinese and Western Medicine Ethical Committee, and informed consent was obtained from all patients. All programs were performed in conformity to the ethical standards of the institutional research committee and in accordance with the 1964 Helsinki Declaration and its latter amendments.

Evaluating the therapeutic efficacy and detecting the concentration of $\mathrm{SH}$ in the patient SF in clinical tests For evaluating clinical efficacy, the test group had nine patients, and the positive control group had six patients. The therapeutic program in the test group was transdermal treatment of SH solution with optimized electroporation combined with standard therapy (eg, celecoxib). Meanwhile, the therapeutic program in the positive control group was only the standard therapy (eg, celecoxib). Clinical efficacy parameters were assessed after 8 days. The parameters for evaluating clinical efficacy were joint pain degree (Visual Analog Scale/Score [VAS]) and joint movement degree. The clinical improvement rate was calculated according to Eq (3). The transdermal treatment method using SH solution with electroporation was as follows: $4 \mathrm{~mL}$ SH solution $(25 \mathrm{mg} / \mathrm{mL}$, injection) was mixed with $5 \mathrm{~mL}$ normal saline and subsequently vortexed. After vortexing to allow homogenization, the mixture was absorbed by two pieces of cotton. The two pieces of cotton containing the liquid mixture were placed in two electrodes, and the two electrodes were subsequently placed on one knee of the patients. Clinical transdermal tests were done using the liquid mixture with electroporation for 30 minutes. The electroporation conditions were as follows: $3 \mathrm{KHz}$, exponential waveform, and intensity 10 .

For detecting the concentration of $\mathrm{SH}$ in the patient SF, the test group had nine patients and the control group had six patients. The clinical transdermal drug delivery in the test group was done using the liquid mixture with electroporation for 30 minutes. The clinical transdermal drug delivery in the control group was done using the liquid mixture without electroporation for 30 minutes. After transdermal administration, the patient SF samples were collected by the hospital staff using the SF collection equipment. The concentration of $\mathrm{SH}$ in the patient $\mathrm{SF}$ samples were determined by an UHPLC-MS/MS system. The transdermal treatment method using SH solution with electroporation was done as described earlier.

\section{Sample preparation for in vitro transdermal experiments}

Samples for in vitro transdermal experiments $(200 \mu \mathrm{L})$ were mixed with $10 \mu \mathrm{L}$ IS solution $(0.5 \mu \mathrm{mol} / \mathrm{L}$ testosterone in methanol) and subsequently vortexed for $5 \mathrm{~min}$. After vortexing to allow homogenization, the mixture was centrifuged at $19,357 \mathrm{~g}$ for $30 \mathrm{~min}$. A total of $5 \mu \mathrm{L}$ of the supernatant layer was injected into the UHPLC-MS/MS column for analysis.

\section{Patient SF sample preparation}

Patient SF samples $(200 \mu \mathrm{L})$ were mixed with $5 \mu \mathrm{L}$ IS solution ( $25 \mathrm{nmol} / \mathrm{L}$ testosterone in methanol) and 1,000 $\mu \mathrm{L}$ methanol solution (5:1), which was added to extract the analytes and remove proteins, and subsequently vortexed for $5 \mathrm{~min}$. After vortexing to homogenize, the mixture was centrifuged at $19,357 \mathrm{~g}$ for $30 \mathrm{~min}$. The supernatant layer was transferred into a new tube and evaporated to dryness in a thermostatic vacuum drier (EYELA Vacuum oven VOS-301SD, Tokyo, Japan). The residues were redissolved using $100 \mu \mathrm{L}$ of $50 \%$ methanol aqueous solution and centrifuged at 19,357 $\mathrm{g}$ for $30 \mathrm{~min}$. A total of $5 \mu \mathrm{L}$ of the supernatant layer was injected into the UHPLC-MS/MS column for analysis.

\section{Analysis and identification of drugs in the receptors}

UHPLC-MS/MS was performed on an Agilent 6540 accuratemass Q-TOF MS system and 6460 Triple Quadrupole mass system with an electrospray ionization source (Agilent Technologies, Santa Clara, CA, USA). The data were recorded, and the system was controlled using the MassHunter software (version B.06.00, Agilent Technologies). The conditions were as follows: column, Zorbax SB-C18, 3.0×100 mm, $1.8 \mu \mathrm{m}$ (Agilent Technologies), with the column temperature maintained at $25^{\circ} \mathrm{C}$; mobile phase $\mathrm{A}, 0.1 \%$ formic acid water; mobile phase $\mathrm{B}, 100 \%$ acetonitrile; flow rate, $0.35 \mathrm{~mL} / \mathrm{min}$; gradient, 0-1.5 min, 15\%-15\% B, 1.5-2 min, 15\%-90\% B, 2-4.2 $\mathrm{min}, 90 \%-68 \% \mathrm{~B}, 4.2-4.7 \mathrm{~min}, 68 \%-15 \% \mathrm{~B}$; and injection volume, $5 \mu \mathrm{L}$.

The main MS/MS working parameters in the positive mode (1-4.7 $\mathrm{min}$ ) were set as follows: $3.5 \mathrm{kV}$ capillary voltage, $500 \mathrm{~V}$ nozzle voltage, $300^{\circ} \mathrm{C}$ gas temperature, $300^{\circ} \mathrm{C}$ sheath temperature, $12 \mathrm{~L} / \mathrm{min}$ sheath gas flow, and $10 \mathrm{~L} / \mathrm{min}$ gas flow. Quantification was performed via the multiple reaction monitoring of the transitions of $\mathrm{m} / \mathrm{z} 330.2 \rightarrow 239.1$ 
and $\mathrm{m} / \mathrm{z} 330.2 \rightarrow 181.0$ for $\mathrm{SH}^{34}$ and $\mathrm{m} / \mathrm{z} 289.0 \rightarrow 97.1$ for IS. Collision energy was set at 26 for SH and 18 for IS. The low limit of quantitative analysis was $0.023 \mathrm{ng} / \mathrm{mL}$ for $\mathrm{SH}$.

\section{Data analysis}

Cumulative drug permeation amount $\left(Q_{n}, \mu \mathrm{g} / \mathrm{cm}^{2}\right)$ was the cumulative amount of drug transdermally delivered in the receiver compartments, and it was computed according to Eq (1).

$$
Q_{n}=\frac{\left[C_{n} \times V+V_{0} \sum_{i=1}^{n-1} C_{i}\right]}{A}
$$

In Eq (1), $\mathrm{A}\left(\mathrm{cm}^{2}\right)$ is the transdermal area, $\mathrm{V}_{0}(\mathrm{~mL})$ is the bulk of samples extracted from the receiver compartments, $\mathrm{V}(\mathrm{mL})$ is the bulk of Ringer's solution in the receiver compartment, and $\mathrm{C}_{\mathrm{i}}(\mu \mathrm{g} / \mathrm{mL})$ and $\mathrm{C}_{\mathrm{n}}(\mu \mathrm{g} / \mathrm{mL})$ are the concentration of sample $\mathrm{i}$ and $\mathrm{n}$.

Steady-state transdermal flux $\left(\mathrm{J}_{\mathrm{ss}}, \mu \mathrm{g} \cdot \mathrm{cm}^{-2} \cdot \mathrm{s}^{-1}\right)$ at time $\mathrm{t}$ represented the slope of the line graphs of cumulative drug permeation amount $\left(Q_{n}\right)$ versus time $(t, s)$. The flux enhancement ratio (ER) induced by electroporation was computed according to Eq (2).

$$
\mathrm{ER}=\left(\frac{\text { Flux with electroporation }}{\text { Flux without electroporation }}\right)=\frac{\mathrm{J}_{\mathrm{ss}+}}{\mathrm{J}_{\mathrm{ss}-}}
$$

Clinical improvement rate was calculated according to Eq (3).

$$
\begin{aligned}
& \text { Clinical improvement rate }= \\
& \frac{(\text { Pretreatment value }- \text { Posttreatment value })}{\text { Pretreatment value }} \times 100 \%
\end{aligned}
$$

Data were represented as mean \pm standard error of mean. One-way analysis of variance was used to evaluate statistical differences. A difference was considered statistically significant at $P<0.05$.

\section{Results and discussion Analysis and identification of $\mathrm{SH}$ in samples}

UHPLC-MS/MS was performed to identify the structure and analyze $\mathrm{SH}$ levels in samples. The results of UHPLC-MS/ MS were as follows: in the representative MRM chromatogram, retention time of $\mathrm{SH}$ was $2.4 \mathrm{~min}$ (Figure 1A) and that of IS was $4.2 \mathrm{~min}$ (Figure 1B); the high-resolution mass spectrometry of SH indicated that the $[\mathrm{M}+\mathrm{H}]^{+}=330.1698$ ion (Figure 1C), and the MS/MS analyses of SH product the $\mathrm{m} / \mathrm{z} 181.0628$ and 239.0674 (Figure 1D). Taken together, the data obtained for the SH in samples was in accordance with the standard substance.

\section{Effect of electroporation parameters and drug concentration on $\mathrm{SH}$ penetration in mice and miniature pig skins \\ Transdermal enhancement by varying electroporation parameters in mice skin}

$1.5,3$, and $6 \mathrm{KHz}$ were chosen to study the effect of pulse frequency on $\mathrm{SH}$ penetration in mice skins. The results demonstrated that the total $Q_{n}$ of $3 \mathrm{KHz}$ was $6.50 \pm 0.94 \mu \mathrm{g} / \mathrm{cm}^{2}$ (Figure 3A1). The ER of $3 \mathrm{KHz}$ was $3.09 \pm 0.41$, which was more than the ER seen at other pulse frequencies (Figure 3A2). Taken together, $3 \mathrm{KHz}$ was found to be the optimal pulse frequency that had the most significant enhancement-promoting effect on SH penetration. Interestingly, we found total $\mathrm{Q}_{n}$ differed in the following manner: $\mathrm{Q}_{\mathrm{n}}$ induced by $3 \mathrm{KHz}>$ $\mathrm{Q}_{\mathrm{n}}$ induced by $1.5 \mathrm{KHz}>\mathrm{Q}_{\mathrm{n}}$ induced by $6 \mathrm{KHz}$. The situation above was exactly opposite to our primary hypothesis that $6 \mathrm{KHz}$ might induce the strongest transdermal enhancement. The reason for this phenomenon might be that $6 \mathrm{KHz}$ produced too many pulses, and $1.5 \mathrm{KHz}$ produced too little pulses, which were not in the optimal range inducing change for skin structure. Thereby, $\mathrm{Q}_{\mathrm{n}}$ induced by $6 \mathrm{KHz}$ and $1.5 \mathrm{KHz}$ were less than that induced by $3 \mathrm{KHz}$, which could produce the exact pulses required for changing the skin structure. To summarize, electroporation did enhance the SH penetration in mice skins, and $3 \mathrm{KHz}$ was the optimal pulse frequency.

Different pulse waveforms produced different $Q_{n}$ in mice skins. The total $\mathrm{Q}_{\mathrm{n}}$ of exponential waveform was $10.48 \pm 1.08 \mu \mathrm{g} / \mathrm{cm}^{2}$ (Figure 3B1). The ER of the exponential waveform was $6.40 \pm 0.64$ (Figure 3B2). That is, exponential pulse produced more intense SH permeation. A previous study showed that exponential waveform was one of the most commonly used pulse waveforms in electroporation..$^{35}$ Generally, increasing pulse amplitude, duration, and number resulted in increased skin permeability, allowing for better drug transport. ${ }^{36}$ The threshold-induced change in the skin structure depends on the pulse duration. An increase in the electronic pulse duration leads to a decrease in the critical threshold, and so pulse duration of exponential pulse was long. ${ }^{37}$ Therefore, we concluded that the exponential waveform was the optimal pulse waveform.

The total $\mathrm{Q}_{\mathrm{n}}$ and $\mathrm{ER}$ at intensity 10 were $18.32 \pm 2.98 \mu \mathrm{g} / \mathrm{cm}^{2}$ (Figure 3C1) and 9.79 \pm 1.69 , respectively, (Figure 3C2), and 

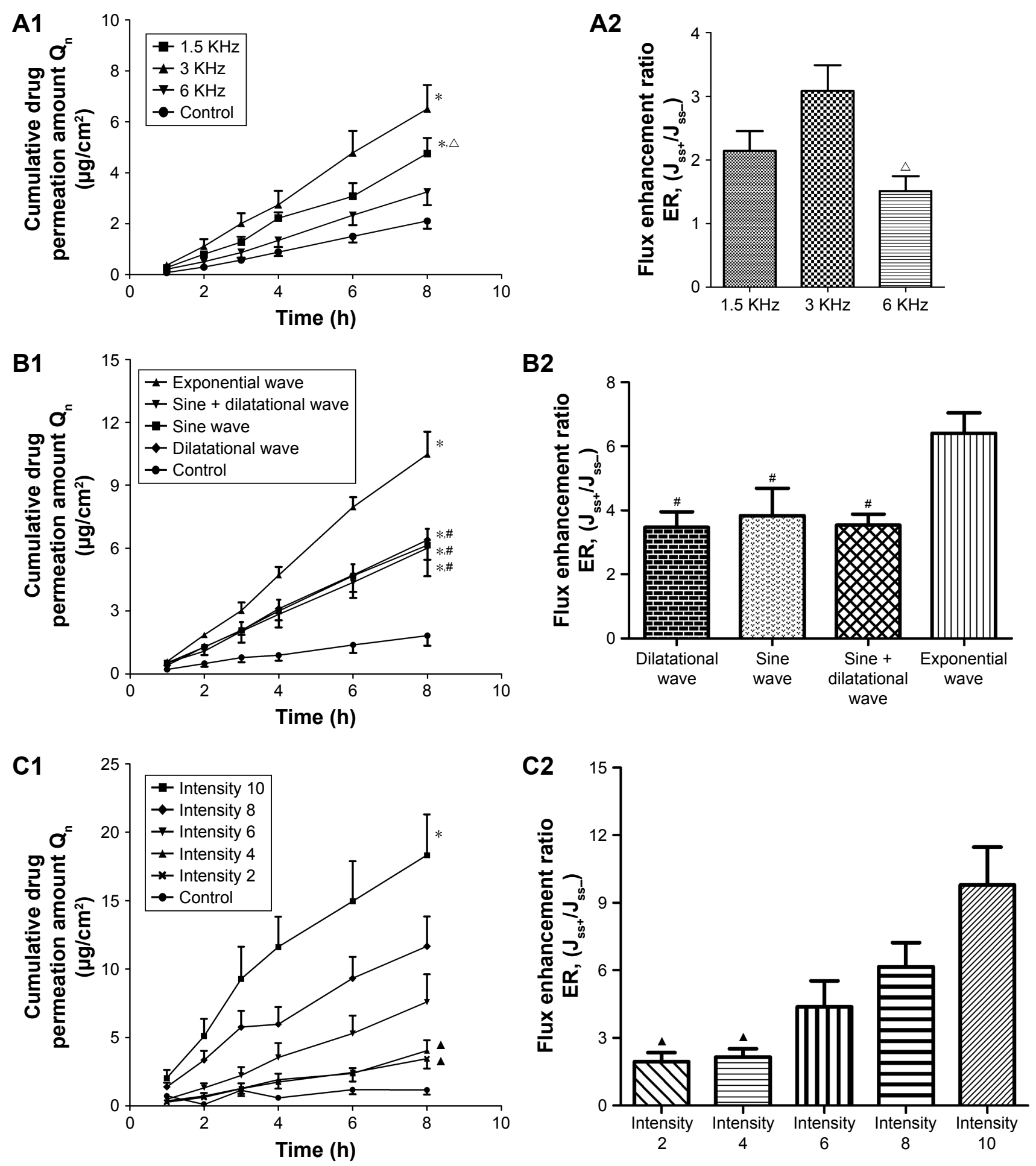

Figure 3 Transdermal enhancement of pulse frequencies, waveforms, and intensity on SH in in vitro mice skins.

Notes: The effect of pulse frequencies on $Q_{n}$-time curves (AI) and ER (A2). The other fixed parameters of electroporation: sine wave and intensity 4. The effect of pulse waveforms on $\mathrm{Q}_{\mathrm{n}}$-time curves (BI) and ER (B2). The other fixed parameters of electroporation: I.5 KHz and intensity 4. The effect of pulse intensities on $\mathrm{Q}_{\mathrm{n}}$-time curves $(\mathbf{C I})$ and ER (C2). The other fixed parameters of electroporation: sine wave and I.5 KHz. $* P<0.05$ versus the control groups. $\triangle P<0.05$ versus the $3 \mathrm{KHz}$ groups. ${ }^{\sharp} P<0.05$ versus the exponential waveform groups. $\triangle P<0.05$ versus the intensity 10 groups. Data are represented as mean $\pm S E M$. $n=6$.

Abbreviations: $\mathrm{SH}$, sinomenine hydrochloride; $\mathrm{Q}_{\mathrm{n}}$, cumulative drug permeation amount; ER, enhancement ratio; SEM, standard error of mean.

$\mathrm{Q}_{\mathrm{n}}$ increased in an intensity-dependent manner in the intensity range $2-10$ (Figure $3 \mathrm{C} 1$ and $\mathrm{C} 2$ ). Therefore, enhanced pulse intensity could enhance skin permeability. For our electroporation equipment, intensity 10 was the maximum intensity that could be given to humans without harm, and for animals it was 4. Using higher intensity might result in excessive stimulation of the animal and make them feel uncomfortable. The total $Q_{n}$ and ER of intensity 10 was more than those seen at 
other pulse intensities. As expected, intensity 10 was found to be the optimal pulse intensity for humans. Above all, pulse frequency, waveforms, and intensity could affect the $\mathrm{SH}$ penetration in mice skins, and the optimal parameters were $3 \mathrm{KHz}$, exponential waveform, and intensity 10 for humans.

\section{Transdermal enhancement of electroporation in miniature pig skins by varying the parameters}

The total $\mathrm{Q}_{\mathrm{n}}$ of $3 \mathrm{KHz}$, exponential waveform, and intensity 10 were $8.67 \pm 0.87,8.67 \pm 0.87$, and $53.92 \pm 5.07 \mu \mathrm{g} / \mathrm{cm}^{2}$, respectively (Figure 4A1, B1, and C1). Meanwhile, ER for $3 \mathrm{KHz}$, exponential waveform, and intensity 10 were $8.12 \pm 0.92,8.12 \pm 0.92$, and $48.17 \pm 3.93$, respectively (Figure 4A2, B2, and C2). These results showed that $Q_{n}$ and ER induced by electroporation parameters $3 \mathrm{KHz}$, exponential waveform, and intensity 10 were higher compared with that induced by other electroporation parameters. On analysing the overall situation, the results in miniature pig skins were in accordance with that in mice skins, and the optimal parameters were also same in both mice and miniature pig skins, and the optimal electroporation parameters were $3 \mathrm{KHz}$, exponential waveform, and intensity 10 for humans. In practical clinical application, we could choose different parameters of electroporation to achieve different kinds of enhancement in transdermal efficiency so as to achieve the purpose of controlled release drugs.

\section{Effect of different drug concentration of $\mathrm{SH}$ on cumulative drug permeation amount in mice and miniature pig skins}

The total $\mathrm{Q}_{\mathrm{n}}$ of different groups were $0.73 \pm 0.08,2.22 \pm 0.67$, $5.41 \pm 1.21$, and $10.57 \pm 2.80 \mu \mathrm{g} / \mathrm{cm}^{2}$ in mice skins (Figure 5A2), and $1.28 \pm 0.35,2.71 \pm 0.71,8.67 \pm 0.87$, and $15.50 \pm 1.01 \mu \mathrm{g} / \mathrm{cm}^{2}$ in miniature pig skins (Figure 5B2). We also found that $\mathrm{Q}_{n}$ increased gradually with the increase of the drug concentration in vitro in mice and miniature pig skin permeation experiments with electroporation (Figure 5A1 and 5B1). According to these results, the total $\mathrm{Q}_{\mathrm{n}}$ of $3.04 \mathrm{mmol} / \mathrm{L}$ was more apparent than that of other drug concentrations in in vitro mice and miniature pig skins. In brief, drug concentration was one of the key factors for electroporation to promote transdermal penetration of $\mathrm{SH}$, and $\mathrm{Q}_{\mathrm{n}}$ values increased in a drug-dependent manner.

Drug penetration routes include intercellular route, intracellular route, and eccrine route (eg, sweat glands). ${ }^{38}$ Cell gap fillers and skin are usually hydrophobic; ${ }^{39}$ therefore, penetrating through the skin into the blood circulation is difficult for the hydrophilic $\mathrm{SH}$. As shown above, the $\mathrm{Q}_{\mathrm{n}}$ of the passive diffusion transdermal experiments without electroporation were all so low that concentration of $\mathrm{SH}$ might not reach the targeted therapeutic concentration. Through inducing the change of skin structure, electroporation could enable $\mathrm{SH}$ to overcome transdermal defects caused by its hydrophilic property. $A 11 \mathrm{Q}_{n}$ values in the four drug concentrations with electroporation were more than that in control without electroporation, and this was a strong proof that electroporation significantly enhanced the transdermal permeation of $\mathrm{SH}$.

\section{Transdermal enhancement mechanism of electroporation in mice and miniature pig skins}

The epidermis is the outermost skin layer that consists of that hypodermis, hydrophilic dermis, and epidermis. ${ }^{40}$ The layers of the epidermis are stratum spinosum, stratum granulosum, and $\mathrm{SC}$, which protects the skin from damage and is the main transdermal barrier of the skin. ${ }^{41}$ On HE staining analysis, the nucleus was blue and the cytoplasm was red (Figures 6A and B, 7A and B). In normal skin tissues, the skin structures were tight and integrated, and the flattened cells of the epidermis were tightly arranged, and the edge of cells was difficult to distinguish (Figures 6A and 7A). After electroporation stimulation, the skin structures were loose, the intercellular intervals and epidermal cracks of skin increased, and the distribution of stratified cells in SC was chaotic (Figures 6B and $7 \mathrm{~B}$ ). Taken together, electroporation did change the skin structure, increased the skin permeability, and enhanced transdermal drug delivery.

NaFluo is a fluorescent agent with a similar structure to SH. The molecular weight of NaFluo is 376.27. SH and NaFluo are both hydrophilic. NaFluo is only distributed in the outmost $\mathrm{SC}$ after passive diffusion without electroporation (Figures 6C and 7C). However, when electroporation was applied to the skins, the stratum spinosum, stratum granulosum, and SC were observed to have very strong fluorescence of NaFluo (Figures 6D and 7D), and this meant that NaFluo deeply permeated into epidermis. ${ }^{42}$ Therefore, electroporation could significantly promote NaFluo transdermal permeation. In summary, the mechanism by which electroporation promoted permeation was that electronic pulses made the skin looser and increased the intercellular spaces in the skin. Also, a schematic mechanism of electroporation enhancing $\mathrm{SH}$ transdermal delivery is shown in Figure 8. In addition, we found that the skin permeability enhanced by electroporation in miniature pig skin was greater than that in the mice skin in this study. This might be because the electroporation thresholds and electrical resistance of miniature pig abdomen skin was smaller than that of skin taken from the backs of mice; thus, the electronic pulse could better penetrate the miniature pig skin and cause the skin structure to change. ${ }^{43}$ 

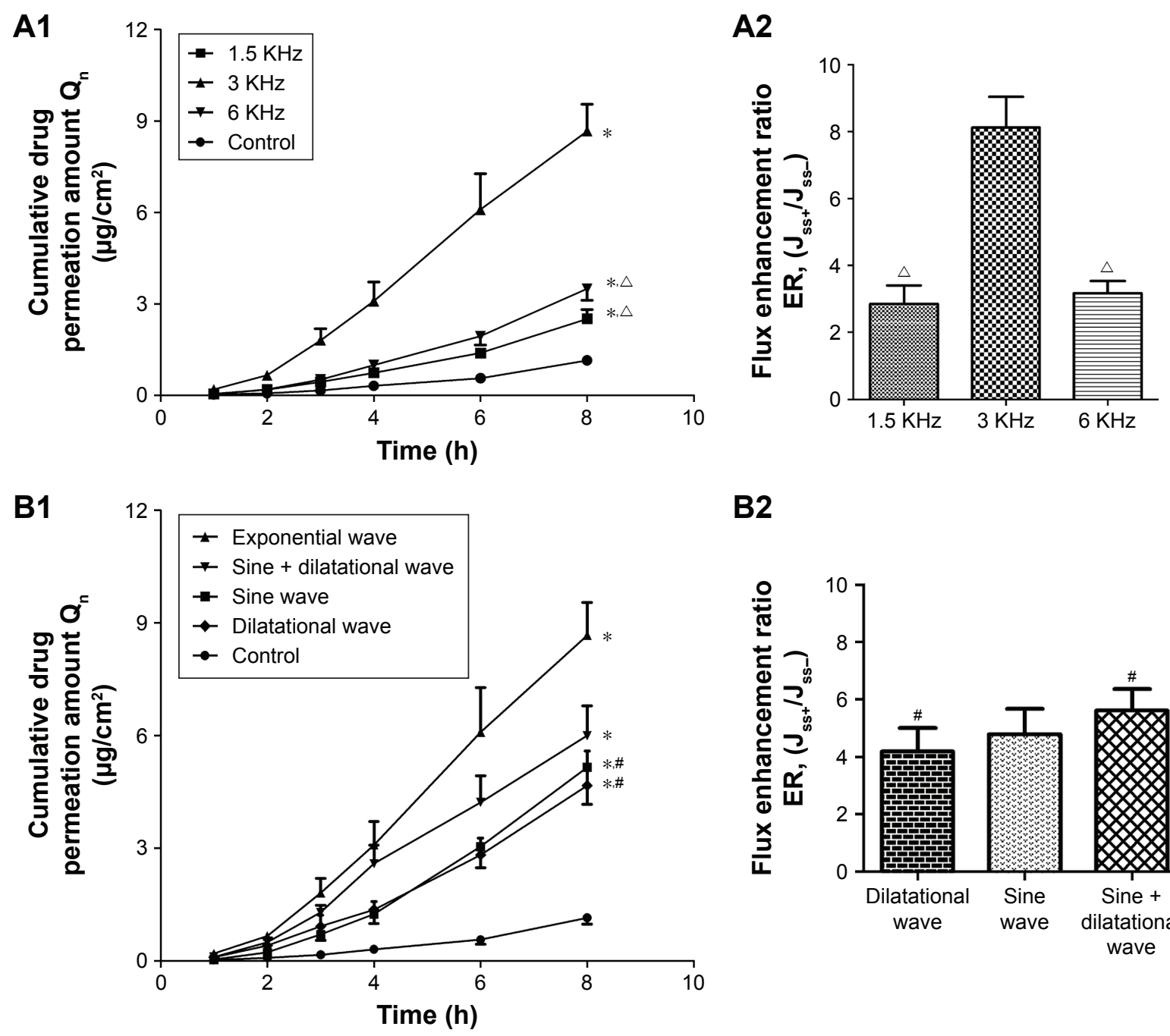

B2
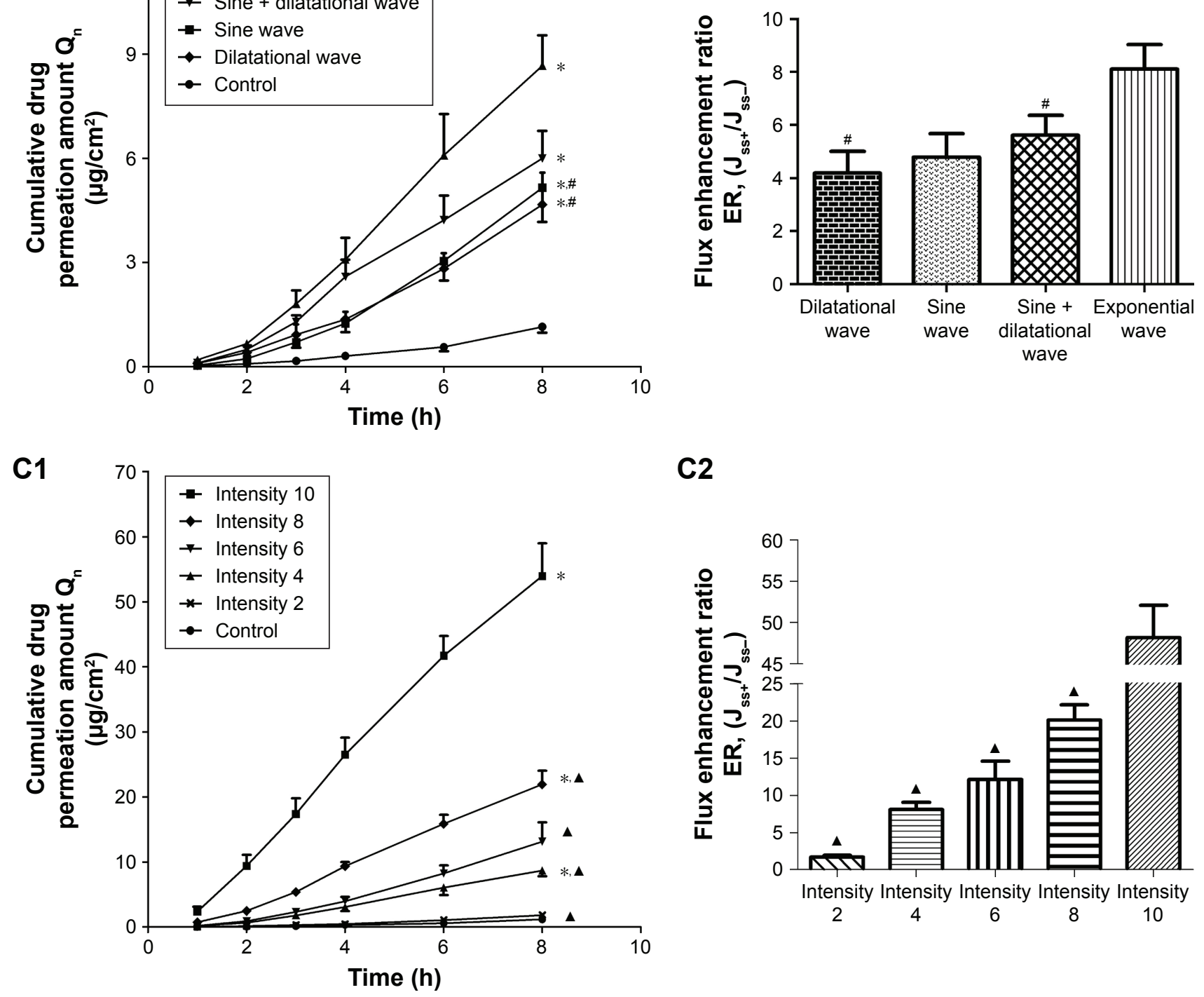

C2

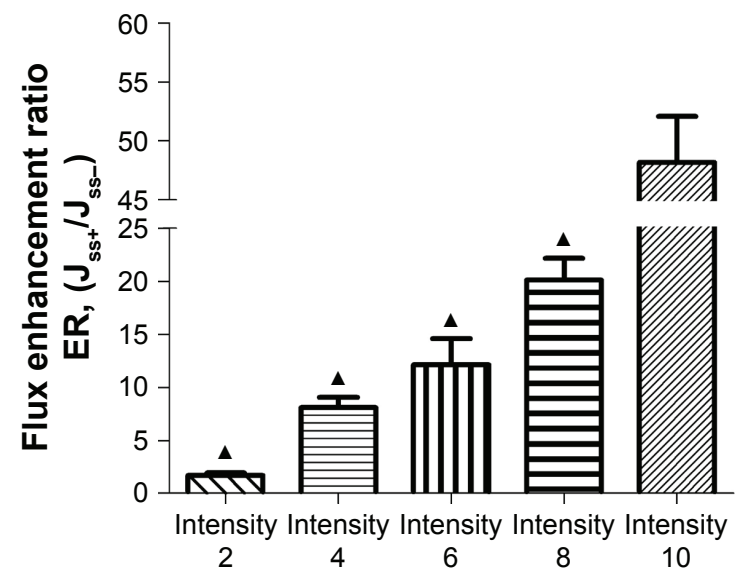

Figure 4 Transdermal enhancement of pulse frequencies, waveforms, and intensity on $\mathrm{SH}$ in in vitro miniature pig skins.

Notes: The effect of pulse frequencies on $Q_{n}$-time curves $(\mathbf{A I})$ and $E R(A 2)$. The other fixed parameters of electroporation are as follows: exponential wave and intensity 4. The effect of pulse waveforms on $Q_{n}$-time curves (BI) and ER (B2). The other fixed parameters of electroporation: $3 \mathrm{KHz}$ and intensity 4 . The effect of pulse intensities on $Q_{n}$-time curves $(\mathbf{C I})$ and $E R(C 2)$. The other fixed parameters of electroporation: exponential wave and $3 \mathrm{KHz}$. $* P<0.05$ versus the control groups. $\triangle P<0.05$ versus the $3 \mathrm{KHz}$ groups. ${ }^{\#}<0.05$ versus the exponential waveform groups. $\triangle P<0.05$ versus the intensity 10 groups. Data are represented as mean $\pm S E M$. $n=6$.

Abbreviations: $\mathrm{SH}$, sinomenine hydrochloride; $\mathrm{Q}_{\mathrm{n}}$, cumulative drug permeation amount; $\mathrm{ER}$, enhancement ratio; $\mathrm{SEM}$, standard error of mean. 

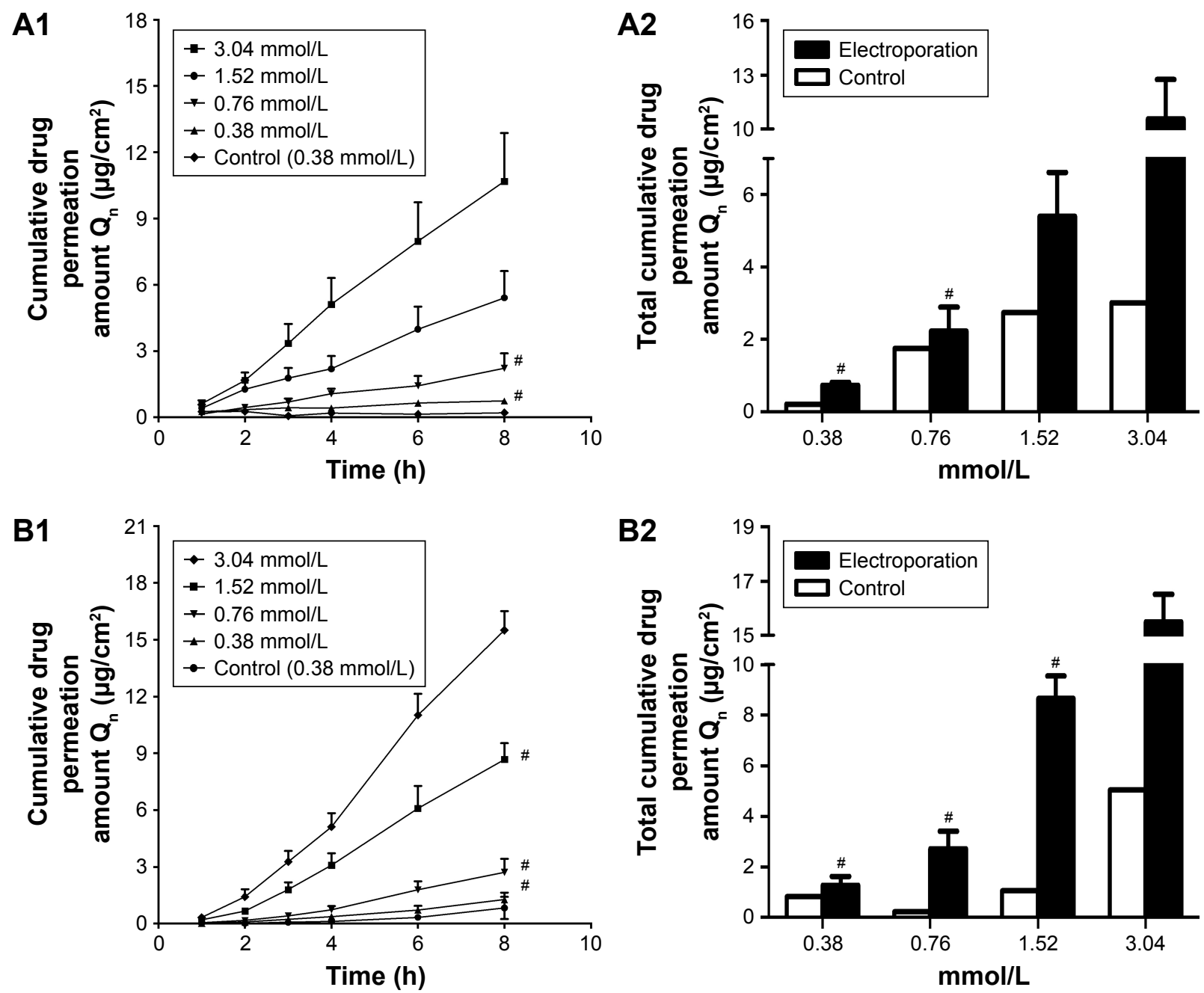

Figure 5 Relationship between drug concentration and transdermal amount of $\mathrm{SH}$.

Notes: The $Q_{n}$ increased as drug concentration rose in the skin taken from the back of mice $(\mathbf{A} \mathbf{I})$ and in miniature pig abdominal skins (BI). Total $Q_{n}$ for different drug concentrations in mice skins (A2) and in miniature pig abdominal skins (B2). Electroporation condition: exponential wave, $3 \mathrm{KHz}$, and intensity 4 . ${ }^{*} \mathbf{P}<0.05$ versus the $3.04 \mathrm{mmol} / \mathrm{L}$ groups. Data are represented as mean \pm SEM. Electroporation groups, $\mathrm{n}=6$. Control groups, $\mathrm{n}=\mathrm{I}$.

Abbreviations: $\mathrm{SH}$, sinomenine hydrochloride; $\mathrm{Q}_{\mathrm{n}}$, cumulative drug permeation amount; $\mathrm{SEM}$, standard error of mean.

\section{Effect of optimized parameter electroporation in clinical tests}

In the test group, the improvement percentage in joint movement degree was $94.75 \% \pm 6.84 \%$ (Figure 9B), and the effective percentage of joint pain relief(VAS) was $79.39 \% \pm 4.63 \%$ (Figure 9C). Compared with the test group, the above two parameters were lower in positive control group, with the improvement percentage of joint movement degree being $52.41 \% \pm 12.51 \%$ (Figure 9D) and the effective percentage of joint pain relief(VAS) being $62.40 \% \pm 7.54 \%$ (Figure 9E). Moreover, the improvement percentage of joint movement degree was statistically significant $(P<0.05)$.

The concentration of SH in the patient SF of the test group and the control group was examined. In the test group, the maximum and minimum concentrations of SH were approximately 20.84 and $0.39 \mathrm{ng} / \mathrm{mL}$, respectively (Figure 9A). In the test group, we found that the concentration fluctuation of SH between different individuals was a little high due to the existence of high personal differences. The concentration of SH in patient SF samples of the control group without electroporation was below limit of quantification, so the results of the control group are not shown in this paper.

Based on the above results, high SH concentration in SF might provide better joint pain relief and joint mobility improvement. Therefore, to ensure therapeutic efficacy, we predicted that the minimum therapeutic concentration of $\mathrm{SH}$ in SF should be $20.84 \mathrm{ng} / \mathrm{mL}$. As previously reported, the maximum concentration $\left(\mathrm{C}_{\max }\right)$ of transdermally delivered sinomenine $(50 \mathrm{mg} / \mathrm{kg})$ with electroporation was $29.33 \pm 4.87 \mathrm{ng} / \mathrm{mL}$ in rabbit plasma and $58.87 \pm 7.50 \mathrm{ng} / \mathrm{mL}$ in rabbit SF, and these were lower compared with oral and injection administration. ${ }^{18}$ However, the $\mathrm{C}_{\mathrm{SF}} / \mathrm{C}_{\text {Plasma }}$ 
A

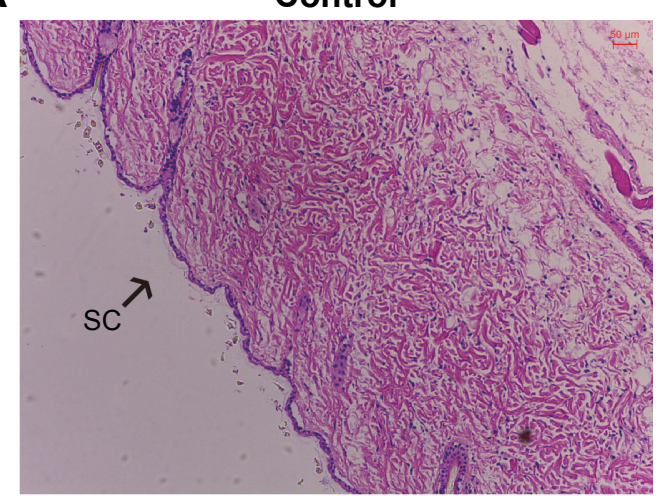

B

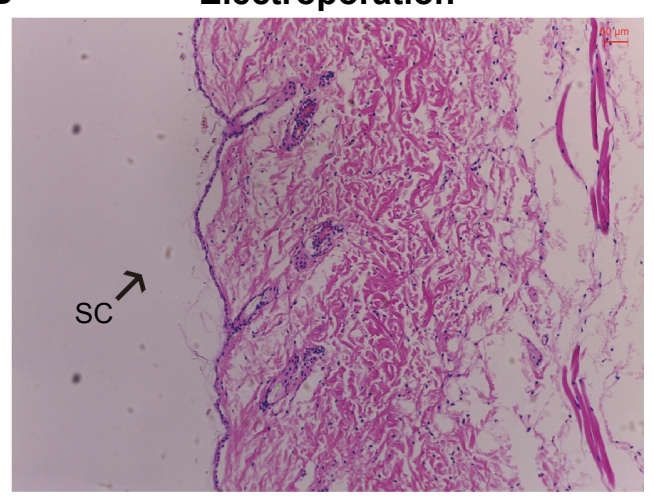

C
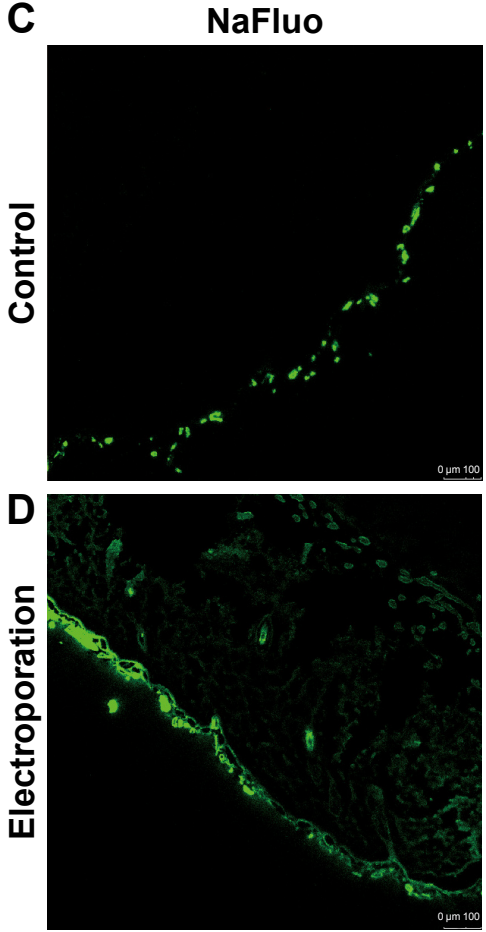

Bright field
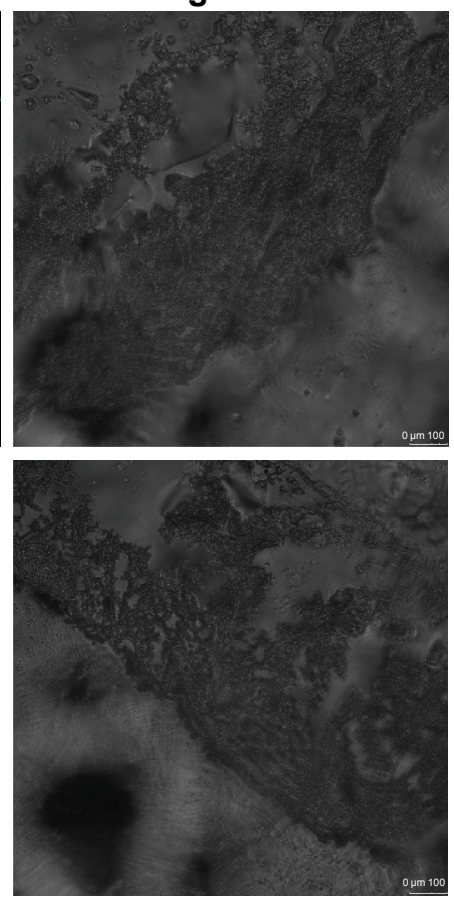
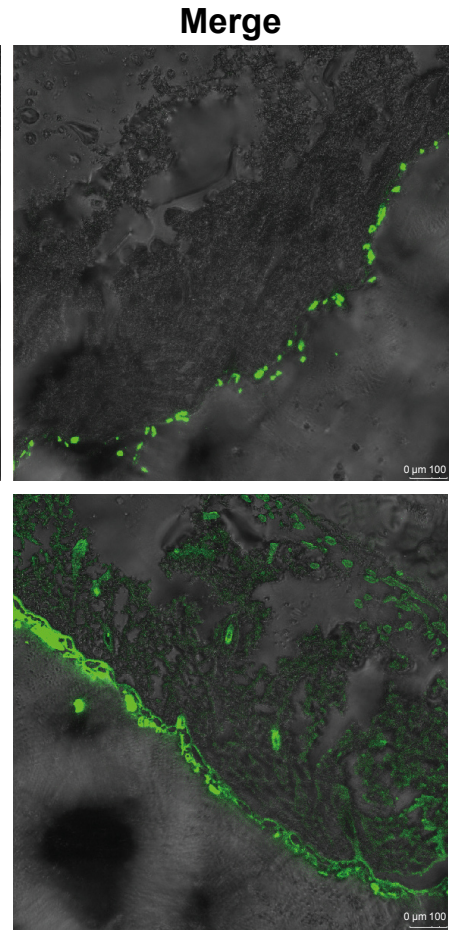

Figure 6 Effect of electroporation on mice skin structure.

Notes: The analysis of HE staining in control groups (A) and in electroporation groups (B). Distribution of NaFluo was in mice skins, and the control and electroporation groups are displayed in (C) and (D), respectively. All experiments were performed in triplicate. Magnification $\times 100$.

Abbreviations: NaFluo, sodium fluorescein; $\mathrm{HE}$, hematoxylin and eosin; $\mathrm{SC}$, stratum corneum.

(concentration of sinomenine in SF versus that in plasma) by electroporation was $3: 1$, and the $\mathrm{C}_{\mathrm{SF}} / \mathrm{C}_{\text {Plasma }}$ of systemic administration routes was approximately 1:3. In this study, the $\mathrm{C}_{\max }$ of transdermally delivered $\mathrm{SH}(11.11 \mathrm{mg} / \mathrm{mL}$, $9 \mathrm{~mL}$, approximately $1.67 \mathrm{mg} / \mathrm{kg}$ ) with electroporation was $20.84 \mathrm{ng} / \mathrm{mL}$ in patient $\mathrm{SF}$. The result revealed that the $\mathrm{C}_{\max }$ of $\mathrm{SH}$ in patient plasma that produced systemic side effects might be low. Hence, the side effects might easily be relieved. Concurrently, high SH concentration in SF could enhance the therapeutic effect of the target joint. In short, transdermal delivery of $\mathrm{SH}$ with electroporation could be selected as a promising treatment regimen of RA and OA to improve efficacy and reduce side effects.
Electroporation has some advantages compared with other transdermal enhancing methods. The chemical transdermal enhancers have some side effects, such as irritation and irreversible skin damage. However, no skin damage is caused in transdermal treatment with electroporation. Another widely used physical means, ie, sonophoresis, is not convenient due to large machines needed and the necessity for skilled nurses. Electroporation is excellent because it uses small machines and is convenient. In general, the major advantages of electroporation on improving transdermal drug delivery include high transdermal efficiency, simple performance, safety, and the small instrument needed. Therefore, electroporation could be 
A

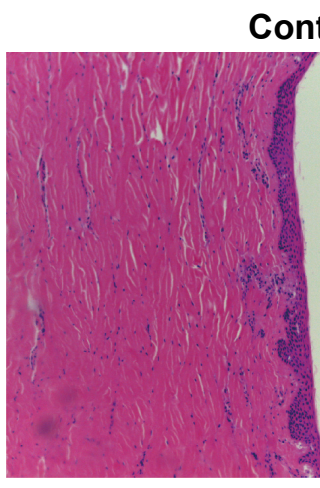

C
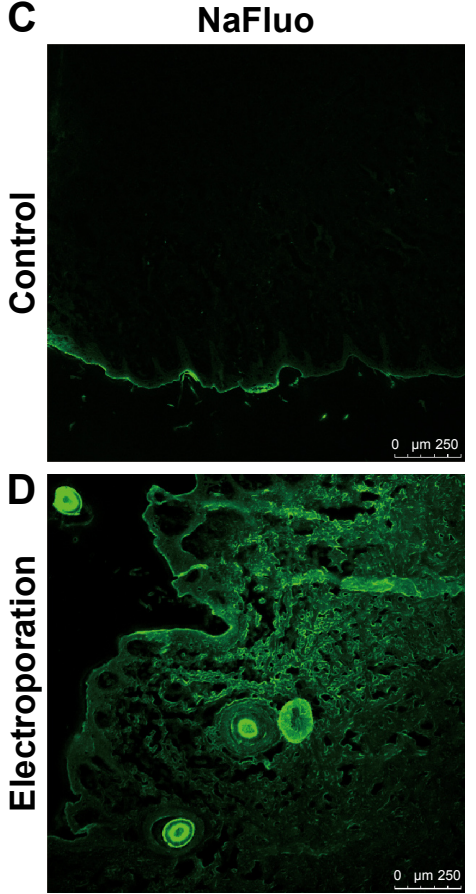

B

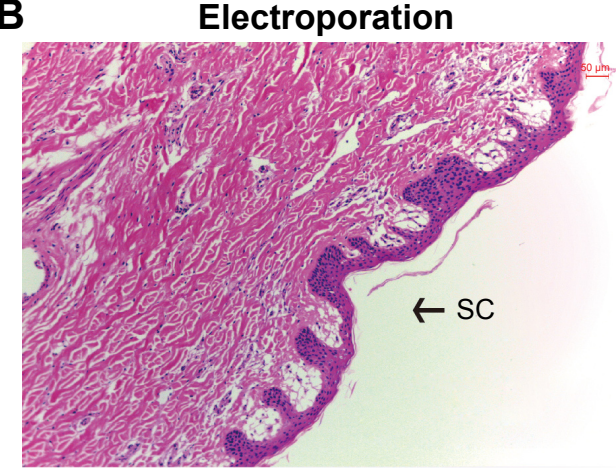

Bright field
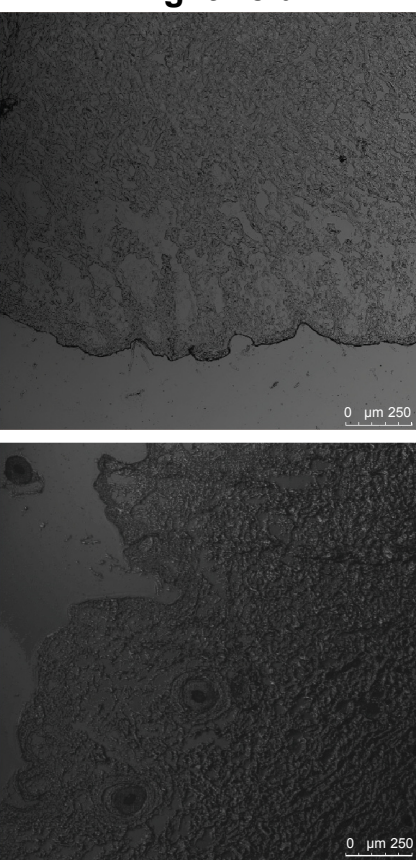
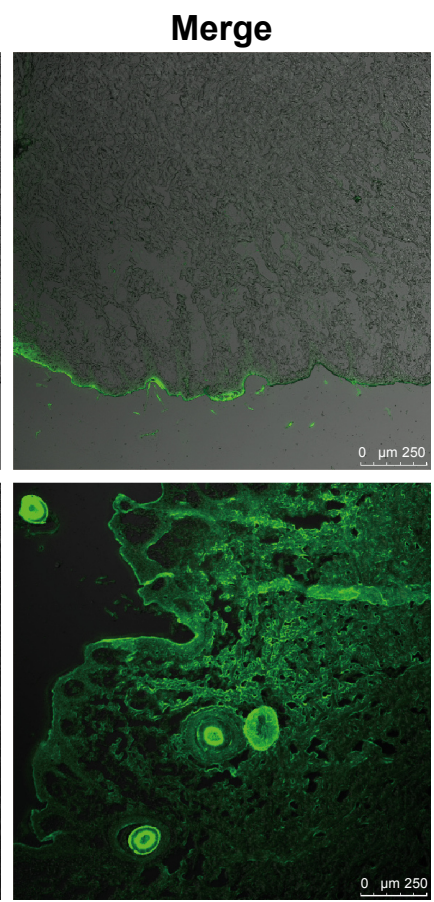

Figure 7 Effect of electroporation on miniature pig skin structure.

Notes: The analysis of HE staining in control groups (A) and in electroporation groups (B). Distribution of NaFluo was in miniature pig skins, and the control and electroporation groups are displayed in (C) and (D), respectively. All experiments were performed in triplicate. Magnification $\times 100$. Abbreviations: NaFluo, sodium fluorescein; HE, hematoxylin and eosin; SC, stratum corneum.

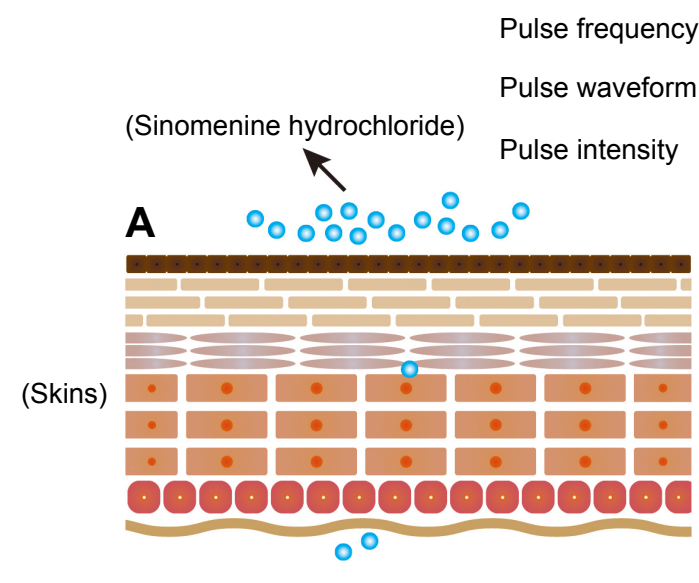

\section{Control}

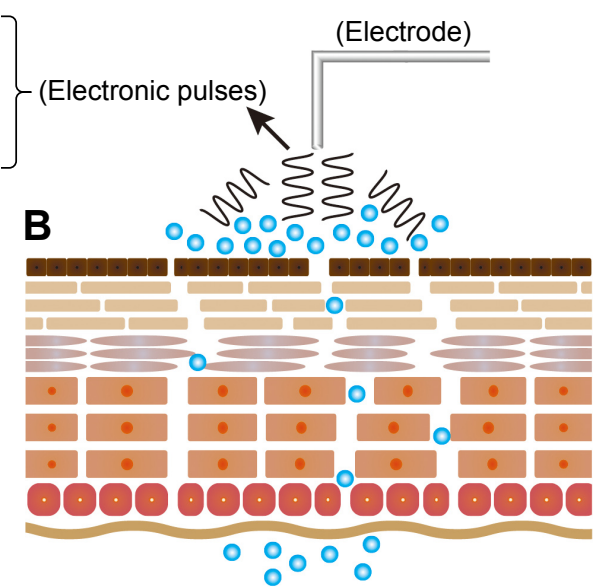

Electroporation

Figure 8 The mechanism of electroporation enhancing SH transdermal delivery.

Notes: (A) In control, the overall skin was tight and integrated. (B) The intercellular intervals and epidermal cracks of skin were increased after the electroporation stimulation, which would result in that $\mathrm{SH}$ had an enhanced permeation in the skin. Abbreviation: $\mathrm{SH}$, sinomenine hydrochloride. 

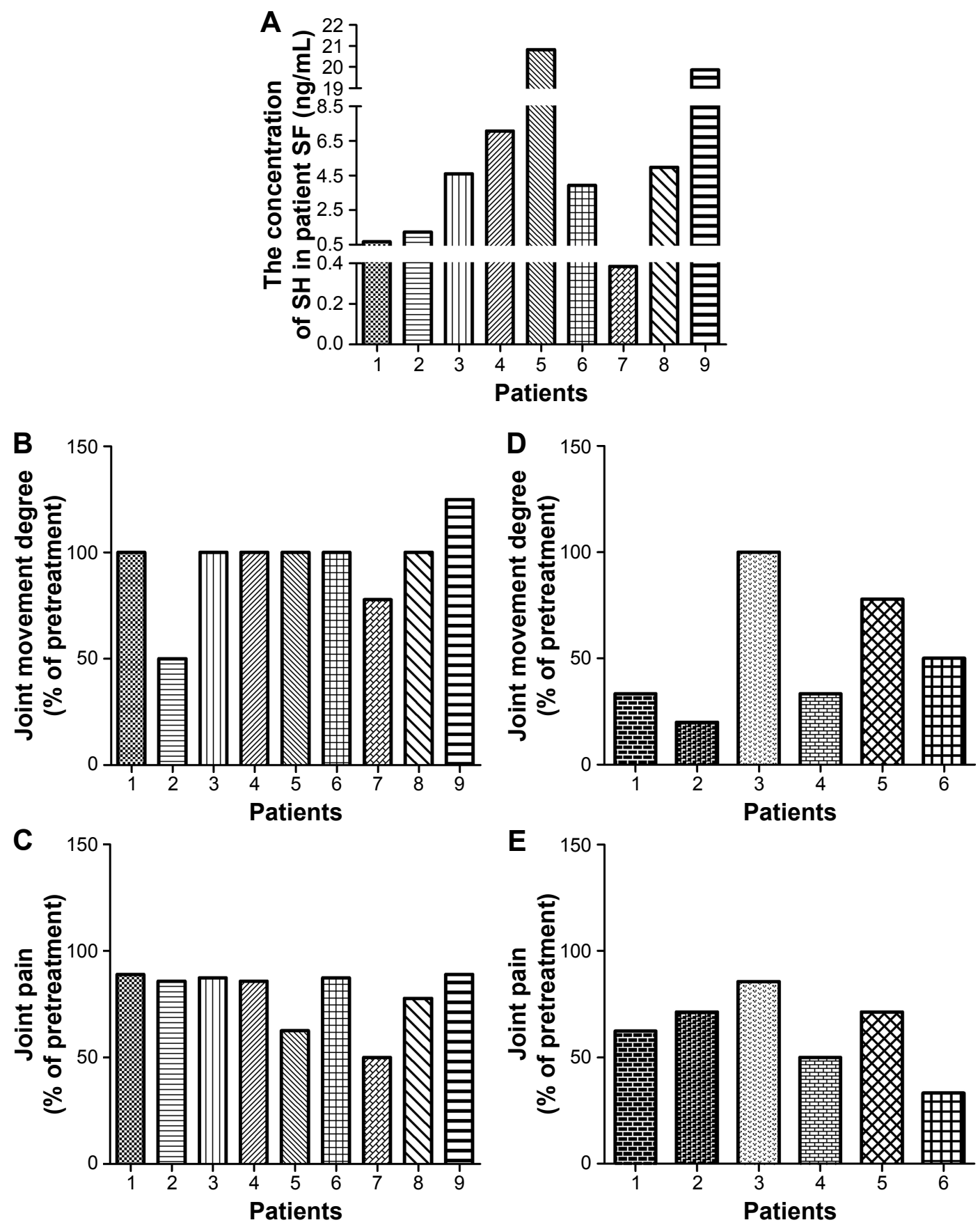

Figure 9 Optimized electroporation increased SH concentration in patient SF and improved the therapeutic effect of SH against arthritis.

Notes: (A) The SH concentration in patient SF of the test group that tested the combination of celecoxib with electroporation. (B) The improvement percentage of joint movement degree in test group. (C) The effective percentage of joint pain relief in test group. (D) The improvement percentage of joint movement degree in the positive control group that was treated with celecoxib without electroporation. (E) The effective percentage of joint pain relief in the positive control group. (B-E) the percentage of clinical symptom improvement is shown as patient self-control. Electroporation condition: $3 \mathrm{KHz}$, exponential waveform, and intensity 10 .

Abbreviations: $\mathrm{SH}$, sinomenine hydrochloride; SF, synovial fluid.

selected for promoting transdermal drug delivery of SH in clinical applications.

\section{Conclusion}

One of the most important factors determining the transdermal enhancement effect of electroporation is the parameters of electroporation. The relationship between electroporation parameters (pulse frequency, waveform, and intensity) and the transdermal enhancement effect of electroporation was deeply investigated in this study. We found that exponential waveform, $3 \mathrm{KHz}$, and intensity 10 were the optimum parameters for electroporation in 
humans. The results of our study demonstrated that with optimized parameters, electroporation exhibited significant enhancing effects on percutaneous permeation of SH. In vitro electroporation-promoting permeation experiments showed that the $\mathrm{Q}_{\mathrm{n}}$ values increased gradually as the drug concentration $(0.38-3.04 \mathrm{mmol} / \mathrm{L})$ increased. Therefore, the drug concentration was also one of the key factors that promoted transdermal penetration of SH by electroporation. The mechanism by which electroporation promoted permeation was that electronic pulses made the SC looser and increased the intercellular spaces in the skins. The electroporation-induced change of skin structure reduced skin barrier properties and promoted $\mathrm{SH}$ permeation. In clinical transdermal test, $\mathrm{SH}$ combined with the optimized parameter electroporation produced high SH concentration in SF. The optimized parameters of electroporation improved the efficacy of $\mathrm{SH}$ through enhancing efficiency of SH transdermal delivery. At the same time, electroporation is safe, uses only small instruments, and is convenient for self-administration by patients. In general, electroporation is an ideal technology for enhancing the transdermal permeation of SH. Electroporation using optimized parameters can be a basis for further improvement of the clinical transdermal application of $\mathrm{SH}$.

\section{Acknowledgments}

This work was supported by the grants from the National Natural Science Foundation of China (81473410 and 81503466), Science and Technology Project of Guangzhou City (201509010004), and Guangdong Natural Science Foundation Province (2015AD030312012 and 2016A050502052).

\section{Disclosure}

The authors report no conflicts of interest in this work.

\section{References}

1. Auger I, Charpin C, Balandraud N, Martin M, Roudier J. Autoantibodies to PAD4 and BRAF in rheumatoid arthritis. Autoimmun Rev. 2012; 11(11):801-803.

2. Pugner KM, Scott DI, Holmes JW, Hieke K. The costs of rheumatoid arthritis: an international long-term view. Semin Arthritis Rheum. 2000; 29(5):305-320.

3. Roczniak W, Brodziak-Dopierala B, Cipora E, Jakobik-Kolon A, Kluczka J, Babuska-Roczniak M. Factors that affect the content of cadmium, nickel, copper and zinc in tissues of the knee joint. Biol Trace Elem Res. Epub 2017 Jan 9.

4. Tacheci I, Bradna P, Douda T, et al. Small intestinal injury in NSAID users suffering from rheumatoid arthritis or osteoarthritis. Rheumatol Int. 2016;36(11):1557-1561.

5. Majima T, Inoue M, Kasahara Y, Onodera T, Takahashi D, Minami A. Effect of the Japanese herbal medicine, Boiogito, on the osteoarthritis of the knee with joint effusion. Sports Med Arthrosc Rehabil Ther Technol. $2012 ; 4: 3$.
6. Bournia VK, Kitas G, Protogerou AD, Sfikakis PP. Impact of nonsteroidal anti-inflammatory drugs on cardiovascular risk: is it the same in osteoarthritis and rheumatoid arthritis? Modern Rheumatol. 2016:1-11.

7. Tacheci I, Bradna P, Douda T, et al. NSAID-Induced enteropathy in rheumatoid arthritis patients with chronic occult gastrointestinal bleeding: a prospective capsule endoscopy study. Gastroenterol Res Pract. 2013;2013:268382.

8. Zhang HC, Liu MX, Wang EP, Lin Z, Lv GF, Chen X. Effect of sinomenine on the expression of rheumatoid arthritis fibroblast-like synoviocytes MyD88 and TRAF6. Genet Mol Res. 2015;14(4):18928-18935.

9. Gao T, Hao J, Wiesenfeld-Hallin Z, Wang DQ, Xu XJ. Analgesic effect of sinomenine in rodents after inflammation and nerve injury. European J Pharmacol. 2013;721(1-3):5-11.

10. Qiu J, Wang M, Zhang J, et al. The neuroprotection of Sinomenine against ischemic stroke in mice by suppressing NLRP3 inflammasome via AMPK signaling. Int Immunopharmacol. 2016;40:492-500.

11. Ju XD, Deng M, Ao YF, et al. Protective effect of sinomenine on cartilage degradation and chondrocytes apoptosis. Yakugaku Zasshi. 2010; 130(8): 1053-1060.

12. Zhang MF, Zhao Y, Jiang KY, et al. Comparative pharmacokinetics study of sinomenine in rats after oral administration of sinomenine monomer and Sinomenium acutum extract. Molecules. 2014;19(8): 12065-12077.

13. Zhao XX, Peng C, Zhang H, Qin LP. Sinomenium acutum: a review of chemistry, pharmacology, pharmacokinetics, and clinical use. Pharm Biol. 2012;50(8):1053-1061.

14. Xu M, Liu L, Qi C, Deng B, Cai X. Sinomenine versus NSAIDs for the treatment of rheumatoid arthritis: a systematic review and meta-analysis. Planta Med. 2008;74(12):1423-1429.

15. Jiang Y, Gao M, Wang W, et al. Sinomenine hydrochloride protects against polymicrobial sepsis via autophagy. Int J Mol Sci. 2015;16(2): 2559-2573.

16. Zhou H, Wong YF, Wang J, Cai X, Liu L. Sinomenine ameliorates arthritis via MMPs, TIMPs, and cytokines in rats. Biochem Biophys Res Commun. 2008;376(2):352-357.

17. Nishida $S$, Satoh $H$. In vitro pharmacological actions of sinomenine on the smooth muscle and the endothelial cell activity in rat aorta. Life Sci. 2006;79(12):1203-1206.

18. Yan H, Yan M, Li HD, Jiang P, Deng Y, Cai HL. Pharmacokinetics and penetration into synovial fluid of systemical and electroporation administered sinomenine to rabbits. Biomed Chromatogr. 2015; 29(6):883-889.

19. Mo ZX, An SL, Zhou JY. [Effects of Caulis Sinomenii and sinomenine on morphine-induced place preference and brain histamine level in mice]. Nan fang yi ke da xue xue Bao. 2006;26(12):1709-1713.

20. Liu ZQ, Chan K, Zhou H, et al. The pharmacokinetics and tissue distribution of sinomenine in rats and its protein binding ability in vitro. Life Sci. 2005;77(25):3197-3209.

21. Wang J, Ruan J, Zhang C, Ye Y, Cai Y, Wu Y. Development and evaluation of the Sinomenine transdermal patch. PakJ Pharm Sci. 2008; 21(4):407-410.

22. Liu X, Chen T, Liu X, Chen Y, Wang L. Penetration effect of ostrich oil as a promising vehicle on transdermal delivery of sinomenine. J Oleo Sci. 2013;62(9):657-664.

23. Nguyen J, Ita KB, Morra MJ, Popova IE. The influence of solid microneedles on the transdermal delivery of selected antiepileptic drugs. Pharmaceutics. 2016;8(4).

24. Kolli CS, Chadha G, Xiao J, Parsons DL, Babu RJ. Transdermal iontophoretic delivery of selegiline hydrochloride, in vitro. J Drug Target. 2010;18(9):657-664.

25. Rangsimawong W, Opanasopit P, Rojanarata T, Panomsuk S, Ngawhirunpat T. Influence of sonophoresis on transdermal drug delivery of hydrophilic compound-loaded lipid nanocarriers. Pharm Dev Technol. 2017;22(4):597-605.

26. Pavselj N, Zorec B, Miklavcic D, Becker S. Experimental factors to be considered in electroporation-mediated transdermal diffusion experiments. J Biomech Eng. 2015;137(12):124501. 
27. Blagus T, Markelc B, Cemazar M, et al. In vivo real-time monitoring system of electroporation mediated control of transdermal and topical drug delivery. J Control Release. 2013;172(3):862-871.

28. Ita K. Perspectives on transdermal electroporation. Pharmaceutics. 2016;8(1).

29. Mohammad EA, Elshemey WM, Elsayed AA, Abd-Elghany AA. Electroporation parameters for successful transdermal delivery of insulin. Am J Ther. 2016;23(6):e1560-e1567.

30. Zhao YZ, Lu CT, Zhang Y, et al. Selection of high efficient transdermal lipid vesicle for curcumin skin delivery. Int J Pharm. 2013;454(1): 302-309.

31. Garland MJ, Caffarel-SalvadorE, Migalska K, Woolfson AD, Donnelly RF. Dissolving polymeric microneedle arrays for electrically assisted transdermal drug delivery. J Control Release. 2012;159(1):52-59.

32. Subongkot T, Pamornpathomkul B, Rojanarata T, Opanasopit P, Ngawhirunpat T. Investigation of the mechanism of enhanced skin penetration by ultradeformable liposomes. Int J Nanomed. 2014;9: 3539-3550.

33. Kijowski R, Blankenbaker D, Stanton P, Fine J, De Smet A. Arthroscopic validation of radiographic grading scales of osteoarthritis of the tibiofemoral joint. AJR Am J Roentgenol. 2006;187(3):794-799.

34. Chen T, Xiang Z, Dong G, et al. An ultra-high performance liquid chromatographic-tandem mass spectrometric method for the determination of sinomenine in human plasma after transdermal delivery of the zhengqing fengtongning injection. Molecules. 2015;20(4):6454-6465.

35. Yarmush ML, Golberg A, Sersa G, Kotnik T, Miklavcic D. Electroporation-based technologies for medicine: principles, applications, and challenges. Ann Rev Biomed Eng. 2014;16:295-320.
36. Pavselj N, Miklavcic D. A numerical model of permeabilized skin with local transport regions. IEEE Trans Biomed Eng. 2008;55(7): 1927-1930.

37. Prausnitz MR, Bose VG, Langer R, Weaver JC. Electroporation of mammalian skin: a mechanism to enhance transdermal drug delivery. Proc Natl Acad Sci U S A. 1993;90(22):10504-10508.

38. Alkilani AZ, McCrudden MT, Donnelly RF. Transdermal drug delivery: innovative pharmaceutical developments based on disruption of the barrier properties of the stratum corneum. Pharmaceutics. 2015;7(4): 438-470.

39. Berko S, Szucs KF, Balazs B, et al. Electroporation-delivered transdermal neostigmine in rats: equivalent action to intravenous administration. Drug Des Devel Ther. 2016;10:1695-1701.

40. Petchsangsai M, Rojanarata T, Opanasopit P, Ngawhirunpat T. The combination of microneedles with electroporation and sonophoresis to enhance hydrophilic macromolecule skin penetration. Biol Pharm Bull. 2014;37(8):1373-1382.

41. Balazs B, Sipos P, Danciu C, et al. ATR-FTIR and Raman spectroscopic investigation of the electroporation-mediated transdermal delivery of a nanocarrier system containing an antitumour drug. Biomed Opt Express. 2016;7(1):67-78

42. Subongkot T, Ngawhirunpat T. Effect of liposomal fluidity on skin permeation of sodium fluorescein entrapped in liposomes. Int J Nanomed. 2015;10:4581-4592.

43. Manda P, Angamuthu M, Hiremath SR, Raman V, Murthy SN. Iontophoretic drug delivery for the treatment of scars. JPharm Sci. 2014;103(6): $1638-1642$
Drug Design, Development and Therapy

\section{Publish your work in this journal}

Drug Design, Development and Therapy is an international, peerreviewed open-access journal that spans the spectrum of drug design and development through to clinical applications. Clinical outcomes, patient safety, and programs for the development and effective, safe, and sustained use of medicines are the features of the journal, which

\section{Dovepress}

has also been accepted for indexing on PubMed Central. The manuscript management system is completely online and includes a very quick and fair peer-review system, which is all easy to use. Visit http://www.dovepress.com/testimonials.php to read real quotes from published authors. 